\title{
Philosophical Interpretation of English Syntax - Semantics - Function Interface Port based on COCA Language Database
}

\author{
Ying An \\ Foreign Languages Department, Binzhou University, Binzhou, 256600, China
}

\begin{abstract}
Keywords: COCA language database; English syntax - semantics - function; interface port; philosophic interpretation
\end{abstract}

\begin{abstract}
Syntax and semantics peculiar to English are difficulties in cognition. In recent years, languages in the range of language database are expanded. Within interface port of functional characteristics, these languages are introduced to preset language database, which is overall orientation of cognitive course. Language database under the structure of COCA clearly distinguishes analysis on word frequency, conventional lexical collocation, word-formation patterns and others within the structure of functional interface. Network framework peculiar to COCA supplies various linguistic materials with true features. Both teachers and students can select suitable translation forms according to their specific interests. Thus, cognitive enthusiasm is mobilized, and old translation approaches are changed. Therefore, it is necessary to make it clear for functional interpretations within the range of philosophy and provide guidance to practice.
\end{abstract}

\section{Introduction}

With the development of science and technology, cognition peculiar to English is gradually emphasized. Language database is provided with auxiliary feature, and it is quite suitable for this subject. Detailed difficulties in English learning include fontal syntactic framework, deeper semantics and associated cognitive function. Emphasis should be laid to the exploration for port obtained by connecting these interfaces. By virtue of searching and programming under microcomputer collaboration, language database peculiar to COCA accelerates the speed of numerical value searching. All sentence patterns obtained by searching highlight distinct representativeness. Under collaboration of language database, it is easier to distinguish deeper connotation concealed in phrases through exploration. Data searching featured by convenience creates a new path for English cognition in the next stage.

\section{A Summary of Relevant Features of Language Database}

In language database within the range of COCA, the number of English vocabulary collected surpasses 0.43 billions. This language database represents a period with relatively large time span. Associated contents include newspapers, magazines and novels. Language database under the framework of COCA is set as the second large language database. It is free of charge for searching in ordinary days. The language database is preset with enough capacity, covering varied language materials. Besides, collected language materials are continuously completed. Proposed searching interface is free of charge for various levels. Above elements decide the fact that the established language database can be regarded as a translation model under auxiliary framework.

Compared with language database under paralleled framework, new language database provided by COCA covers resources in multiple levels. At the same time, it represents the feature of bilingual parallel, with irreplaceable value. COCA is set as written materials unique to source language. Collaborated with translation lessons at ordinary days, it can be also used to search microcosmic cognition of language database collected and add connotation to lessons.

Proposed classroom design and implementation in the next stage can rely on established language database to input preset target language database. Input language is set as accepted target language. Through path conversion given by COCA, original language can be changed into language framework under particular literary form, and then displayed. This process avoids 
conventional translational processing, and restores features of original English. Linguistic cognition under COCA collaboration encourages students to accept various unprocessed language materials. Thus, it boosts task-based teaching under drive framework. At the same time, students, through careful reflection, distinguish linguistic rules in higher levels instead of staying simple imitation in the original stage.

Language database unique to COCA can distinguish available vocabulary within vocabulary with approximate features, and screen the vocabulary in the next stage, so as to select a suitable translation style. Thus, it can be seen that new paragraphs translated via COCA improve the original accuracy, and promote the improvement to translation speed.

\section{Various Ports under Philosophical Framework}

COCA creates English cognition with autonomous features, and gets rid of conventional guidance to lessons. In spontaneous cognition, students autonomously make exploration, and check preset syntax and semantics. It represents independence feature in cognition. Specifically, interface port covered in the language database can be divided into the following levels:

(I) Fontal semantic searching

In the unique flow of English cognition, students lay emphasis on distinguishing various phrases within the range of contents. Lexical semantics covered in phrases can be divided into positive semantics and passive semantics. Semantics of positive words can be easily remembered through activation. Although semantics of passive words are distinguished, it is very difficult to be accepted or applied. Fontal English semantics reflect given standard of subjective cognition. In this range, the optimal pattern selected is activated passive semantics through vocabulary practices in multiple levels. They are changed into language easily to be accepted.

For instance, this application under auxiliary framework represents the following flows: within the unique range of English words, lots of conventional words are included, all of which contain the character "e. While checking unique vocabulary in this range, many semantics can be searched. Thus, it is convenient for semantic distinguishing in ordinary days, letting students to be clear about different meanings at multiple levels. By virtue of language database under the integration state, concealed meanings of passive words can be activated, so as to promote the improvement to expression level.

(II) Syntactic checking

Interface matched with COCA is provided with online interface under the framework of text analysis. In the proposed interface, a given paragraph can be output. Enough associated information can be obtained by clicking preset search button. All words searched are associated with this fontal syntax. Fundamental meanings of auxiliary features, conventional matching paths, given indexes and word frequency obtained by syntax statistics can be obtained by clicking a certain statement in the paragraph. Besides, other associated information can be also distinguished.

To be specific, fontal searching results can be obtained by inputting certain syntax in the paragraph into proposed box in the upper part of the interface. Attached syntax resources can be obtained by clicking certain sentences in corresponding framework according to conventional analysis needs. Then, teachers can rely on given syntax sequence to analyze syntax connotation at various levels.

For instance, certain conventional syntax covers unique sentences related to "size". The usage under auxiliary framework is conventionally set as the following pattern: size + of. Students can search and select conventional phrases under auxiliary orientation, such as shoes and pen. According to represented paragraph connotation, students can screen and select accurate syntax under matching state. If unique meaning of the word "running water" needs to be represented, conventional sentence construction covers fontal usage of "water" as well as new usages with emotional orientation. Language database unique to COCA can distinguish particular application frequency of various usages. As shown in searched results, the conventional syntax of water can be used to describe a certain object with objective features, but such description lacks emotional coloring in a higher level. According to proposed practical context and corresponding to translated 
materials, sentence construction with emotions is accepted, complying with graceful implication concealed in the contents.

\section{(III) Functional checking of auxiliary features}

Higher level in cognition is set as paragraph function under integrated framework. Language database matched with COCA can distinguish fontal function, analyze concealed pattern of paragraph, extract associated words obtained, and represent reasonable orientation in a higher level. If proposed contents are to be translated, unique words obtained through searching can be used to distinguish accuracy unique to function and appropriate degree under auxiliary framework. For this search flow, it is necessary to be provided with new train of thought as well as searching flows under the state of multiple dimensions. Thus, enough information can be mastered. Selected translation path can highlight the feature of appropriateness and optimality.

For instance, paragraphs representing the meaning of "atmosphere" are conventionally matched with cognition within the range of geography. Most associated nouns under auxiliary state represent geographic objects peculiar to a certain region. If selected contents should be set as simple and unsophisticated atmosphere of certain scene, the optimal matching searched should be integrated with atmosphere within the range of "ancient" and "historic". According to careful screening, above matching frameworks correspond to concealed function of paragraph. However, the atmosphere of translation created by "historic" is closer to the function of the original contents, reflecting unique plain atmosphere of village. Such function and matching frequency are set as a large range. Through above exploration, the function of summarized contents can be realized.

Unique application of language database at ordinary days can also collaborate with students to ascend from word investigation in the initial stage to integrated port in a higher level. For instance, students conventionally translate a proposed proverb according to a rigid path, which fails to highlight connotation of fontal statement. Language context described by COCA should be widely accepted. Proverbs and certain associated mottos are of low frequency of being searched. However, these proverbs and mottos are frequently applied. Identification degree unique to proverb and motto is closely associated with flexible application in the next stage. In COCA, the application framework with activation feature is preset, providing usages and paths under flexible state.

\section{New Attributes Unique to COCA}

(I) Identify various word frequencies

English phrases cover various word frequencies. While reading phrases, students will continuously find new associated words. Under this circumstance, how to identify varied words is a dominant difficulty to be solved. The list of new words fails to highlight emphasis. Therefore, students pay too much attention to distinguish complex phonetic rules and new uncommon words, which hinders the improvement to cognitive speed. To reduce cognitive difficulty, a word system under multi-level framework is preset in COCA. There is no inevitable correlation between words with high frequency and preset cognitive emphasis. However, proposed words with particular emphasis conventionally represent higher frequency. The interface created can be used to preset distribution of time in cognition, collecting cognition with much attention to preset words with particular emphasis. Pages segmented by COCA include particular searching of word frequency. The number of conventional words collected in these pages is 60,000.

For instance, frequency words input in COCA is set as peculiar vocabulary of "change". At up right corner of the interface, frequency results obtained can be clearly searched. Results obtained by searching represent parts of speech segmented in detailed from "change", integrating nouns and verbs. Within the range of noun and verb, it highlights higher frequency of application. When used as a verb, this word is of spoken feature. "Change" in the feature of a noun is conventionally used in presentations in a written form. Compared with verb, therefore, this word is featured by the orientation of a noun, highlighting more formal orientation.

(II) Construct multiple correlations 
Diversified words collected in the language database are different in matching. However, these words in different ranges conceal close correlation. Connection under a certain category trends to usage framework under abstract state. Detailed matching form is of segmented pattern peculiar to connection. Varied matching of words includes conventionally mentioned expletive notional words and words under the framework of diversified parts of speech. COCA fails to highlight colligation with visual feature. Cases obtained by searching will be further summarized as concealed rules. Correlated matching patterns can be remaindered through given lists or selected cases.

For instance, words with particular meaning of "invitation" are conventionally used to match with preset subjects and corresponding gatherings. Given searching conditions can be typed into certain region preset on the left of the page. After defining specific conditions, various words can be input into the list under default state. Then, COCA will highlight matching words under matching state. Multiple results obtained through searching contain new matching patterns and varied information under a certain circumstance. MI preset in COCA represents the closeness of correlation. Branch results corresponding to "invitation" can be used to distinguish received invitation from rejected invitation. According to the results searched, the mode of application can be clearly selected.

\section{Conclusion}

The language database integrates proposed normal forms of usage, for the convenience of deviation correction in cognition flow. Language database under the category of COCA can be used to cultivate independent ability at a higher level, and enrich existing cognitive connotation. Diversified materials collected by COCA still have limited disadvantages. English usage under the most suitable framework should be selected with great attention. Syntax \& corresponding semantics and potential functions of paragraph are contained in this language database. Smooth expression is added to new function under philosophical interpretation, promoting the improvement to cognitive effect.

\section{References}

[1] Zhu Xiaomin, Exploration for Translation Teaching based on COCA Language Database and CCL Language Database [J], Foreign Language Teaching, 2011 (01);

[2] Fang Ling and Wang Xingfu, Autonomic Learning and Application of COCA (Corpus of Contemporary American English) [J], Chinese Foreign Language, 2010 (06);

[3] Shao Hong, Application of COCA in English Translation Teaching of Chinese Tourism Texts [J], The World \& Chongqing (Academics), 2014 (04);

[4] Liu Xi, Research on New Mode of College English Vocabulary Teaching based on COCA [J], Journal of Southwest Agricultural University (Social Science Edition), 2013 (12);

[5] Wang Guan, Exploration for and Analysis on College English Vocabulary Teaching based on COCA [J] College English (Academic Edition), 2013 (01);

[6] Wang Yajun, Translation of English Culture Characteristic Words based on COCA [J], Journal of Longdong University, 2014 (04);

[7] Ma Naqin, Research on Stylistic Teaching based on COCA [J], Journal of Chifeng University (Philosophy and Social Science), 2014 (03);

[8] Hui Jinglu, Research on Characteristics of English Proverb Variants based on COCA [J], Journal of Harbin University, 2012 (07);

[9] Wang Dapeng, Analysis on English Synonyms based on COCA - with Synonyms for “Obtain” as an Example [J], Journal of Bohai University (Philosophy and Social Science Edition), 2013 (01); [10] Xue Xiaojuan, Research on Synonyms for "Confused" and "Puzzled” based on COCA [J], Overseas English, 2013 (18). 